Classification

Physics Abstracts

$81.30 \mathrm{~F}-64.70 \mathrm{D}$

\title{
On the stability of low anisotropy dendrites
}

\author{
W. van Saarloos $\left({ }^{1}\right)$, B. Caroli $\left({ }^{2,}{ }^{*}\right)$ and C. Caroli $\left({ }^{2}\right)$ \\ (1) Instituut Lorentz, University of Leiden, P.O. Box 9506, 2300 RA Leiden, The Netherlands \\ (2) Groupe de Physiques des Solides, associé au CNRS, Universités Paris VII et Paris VI, 2 Place \\ Jussieu, 75251 Paris Cedex 05, France
}

(Received 17 September 1992, revised 2 November 1992, accepted in final form 2 November 1992)

\begin{abstract}
Résumé. - Nous montrons que, si on considère la croissance des branches d'une dendrite comme l'invasion du cristal aciculaire (localement instable vis-à-vis du mécanisme de Mullins-Sekerka) par un front de déformation, on prévoit, dans la limite des faibles anisotropies capillaires, que les branches peuvent, au moins transitoirement, remonter vers la tête de la dendrite. Nous montrons que ce point de vue est compatible avec les descriptions à la Zel'dovich de l'évolution des perturbations. Nous proposons une méthode pour tester expérimentalement l'importance de ces effets.
\end{abstract}

\begin{abstract}
We point out that the growth of dendritic sidebranches, when viewed as the propagation of a front into the Mullins-Sekerka unstable needle crystal profile, leads one to expect sidebranches to encroach on the tip, at least transiently, in the limit of small kinetic or surface tension anisotropy. We show that this view point can be reconciled with the Zel'dovich-like description of perturbations and suggest how the importance of these effects for real dentrites could be probed experimentally.
\end{abstract}

\section{Introduction.}

Most recent theoretical work on dendrites (for reviews, see e.g. Refs. [1]-[3]) is based on the idea that the characteristics of freely growing dendrites can be analyzed in two steps ( ${ }^{1}$ ). The first step is to solve the steady state needle crystal problem. In the fully isotropic system no steady state solution exists. Surface tension or kinetic anisotropy, however small its amplitude, results in the existence of a discrete set of steady state solutions with particular values of the growth velocity $V$ and the tip radius of curvature $\rho$. In the second step, as was first done for the

$\left(^{*}\right)$ Also at Département de Physique, Faculté des Sciences fondamentales et appliquées, 33 rue StLeu, 80000 Amiens, France.

(1) This program has been implemented up to now only in two dimensions, and, unless noted otherwise, we will in this paper focus on the two-dimensional problem as well. 
Saffman-Taylor problem [4], one studies the linear stability of these steady state solutions. It is found that the linear spectrum can be separated into two parts : a set of discrete tip-splitting modes which make all but the fastest growing steady state solution unstable, and a continuous spectrum. While the discrete tip-splitting modes have been obtained analytically for small surface tension anisotropy [5], [6], the continuous spectrum has so far only been studied numerically [7], and was found to be stable. Numerical accuracy limits such numerical studies to investigating values of the surface tension anisotropy which are not too small. Since, according to this analysis, there exists one and only one steady state solution which is linearly stable, this, in principle, determines the growth velocity and radius of the Ivantsov parabola describing the tip profile.

The occurrence of sidebranching has been studied in the context of the linear stability of the needle crystal. The sidebranches are usually interpreted as resulting from the combined effect of the selective amplification of noise from the tip region and the advection of the perturbations away from the tip ( ${ }^{2}$ ). This scenario, first proposed by Zel'dovich et al. [9] for flames on a heuristic basis, has been given analytical support by several studies of the late stage of the evolution of a localized perturbation [3], [10], [11]. These investigations focus on the description of the central region of the wave packet describing the perturbation. Since this central region is always found to move away from the tip, it has been concluded that the corresponding amplitude of the perturbation close to the tip must necessarily decrease exponentially in time. This then precludes the existence of oscillating dendrites with perfectly coherent sidebranches.

The above scenario for the origin of sidebranches poses the following question : could the spreading and growth of the wave-packet be fast enough that the corresponding amplitude of the profile deformation in the tip region could increase ? Even if this would occur only transiently, one would have to investigate two possibilities for this description to break down : either a Hopf bifurcation in the linear spectrum might occur, resulting in a coherently oscillating dendrite, or the system could cross over in the tip region to the nonlinear regime, locking into a tip-splitting or oscillating regime.

We expect the above question to be particularly relevant in the limit of small anisotropies, since in this limit the solvability parameter $\sigma \equiv d_{0} \ell / \rho^{2}$ becomes arbitrarily small [1]-[3]. Here $d_{0}$ is the capillary length and $\ell=2 D / V$ the diffusion length, with $D$ the thermal diffusivity. A very small value of $\sigma$ means that the Mullins-Sekerka length scale $\lambda_{\mathrm{MS}} \equiv 2 \pi \sqrt{d_{0} \ell}$ is much smaller than the tip radius, and hence the timescale $2 \pi \sqrt{d_{0} \ell} / V$ for the amplification of perturbations becomes much shorter than the timescale $\rho / V$ for a perturbation to feel that it is advected along a curved interface. Therefore, one at least intuitively expects that, in the limit of small $\sigma$, the growth of the wave-packet might overcome the stabilizing effect of advection. Such a dynamic instability, if present, would compete with the mechanism recently proposed by Esipov [12] to destroy the stable dendrite tip.

In this article, we investigate this question in more detail from two different angles. One approach is to consider the spreading of the sidebranches towards the tip as the propagation of an envelope front into the Mullins-Sekerka unstable flanks of the needle crystal profile. From this we predict that for small $\sigma$, the region near the tip (i.e. whose interface normal makes an angle $\theta$ less than $60^{\circ}$ with the growth direction of the tip) is unstable against the invasion of a sidebranch front. We then show that this result is consistent with the predictions of the wavepacket analysis. We have tried to investigate whether this could result in a Hopf bifurcation in the linear spectrum of the needle crystal for small $\sigma$; as we will discuss, however, the

(2) While most recent work has focussed on this scenario, others have been proposed as well - see e.g. Martin and Goldenfeld [8] for a discussion of various alternative mechanisms. 
approximations which allow one to study the tip splitting spectrum analytically can not be extended to a study of neutral oscillatory modes. As a result, we can not make definite predictions but can only point to their possible occurrence in materials with small anisotropy and sketch some likely consequences if this would happen. We argue that a systematic experimental study of the spatial growth rate of the sidebranches close to the tip would permit to estimate the practical range of applicability of our description, and hence its possible relevance to experimental situations.

\section{Sidebranch evolution as a front propagation problem.}

Let us consider a dendrite of a weakly anisotropic material growing with a small value of $\sigma$. We imagine that a localized perturbation of the needle crystal profile has started developing according to the Zel'dovich scenario, and that it has grown sufficiently large that the perturbation in the tails has reached an observable level. We now concentrate on the tail of the packet on the side closest to the tip. Since in the small anisotropy limit the radius of curvature of the unperturbed profile is much larger $\left({ }^{3}\right)$ than the Mullins-Sekerka length $\lambda_{M S}$, it seems reasonable to view the spreading of the tail of the packet as the propagation of a sidebranch front into the locally planar and therefore Mullins-Sekerka unstable profile. Recent work on such front propagation problems by various authors [13]-[15] has led to the conclusion that the speed of propagation $v$ of such a front satisfies

$$
v \geqslant v^{*}
$$

Here $v^{*}$ is the so-called linear marginal stability velocity which can be calculated explicitly from the dispersion relation $\omega(k)$ of the linearly unstable state into which the front propagates. More precisely, $v^{*}$ is the velocity at which the group and envelope velocities of the front are equal, and is given by

$$
v^{*}=\frac{\partial \omega_{r}}{\partial k_{\imath}}=\frac{\omega_{r}}{k_{\imath}}, \quad \frac{\partial \omega_{\imath}}{\partial k_{r}}=0
$$

where we have used the convention $\left({ }^{4}\right) \mathrm{e}^{\omega t+i k x}$ in considering a perturbation. Here $\omega=\omega_{r}+i \omega_{1}$ and $k=k_{r}+i k_{l}$. In most situations, $v^{*}$ defined by (2) is actually the asymptotic front velocity. Larger front speeds can occur essentially as a result of one of two effects : (i) special initial conditions; and (ii) nonlinearities. In the analysis below, we assume that the actual asymptotic front velocity equals $v^{*}$.

For the Mullins-Sekerka instability of a plane growing with a normal velocity $v_{n}$, the dispersion relation is [16]

$$
\omega=v_{n} k\left(1-d_{0} \ell_{n} k^{2}\right),
$$

where $\ell_{n} \equiv 2 \mathrm{D} / v_{n}$ is the diffusion length corresponding to this normal velocity. This gives with (2)

$$
\begin{aligned}
& k_{r}^{*}=\sqrt{\frac{1}{2 d_{0} \ell_{n}}}, \quad k_{i}^{*}=\sqrt{\frac{1}{6 d_{0} \ell_{n}}}, \\
& v^{*}=\sqrt{3} v_{n} .
\end{aligned}
$$

${ }^{3}$ ) E.g., if there is a surface tension anisotropy $\varepsilon, \rho$ scales at a fixed undercooling as $\varepsilon^{-7 / 4}$ in two dimensions.

$\left({ }^{4}\right)$ Our definition of $\omega$ differs by a factor $-i$ from the one used in reference [15]. This is done to conform to standard useage in the analysis of the Mullins-Sekerka instability, and to the notation used in reference [11], on which our discussion in the next section will be based. 
Note that $k_{\imath}^{*}$ defines the spatial growth rate of the front envelope, and $k_{r}^{*}$ the local wave vector; hence the shape of the front profile in the leading edge is completely specified.

We now apply these results to describe the spreading of the sidebranches in a region of the needle crystal whose normal makes an approximate angle $\theta$ with the growth direction - (see Fig. 1). In this case $v_{n}=V \cos \theta$, and hence the tangential spreading velocity $v_{s b}^{t}$ along the interface is given by equations (2) as

$$
v_{s b}^{t}=v^{*}=\sqrt{3} V \cos \theta
$$

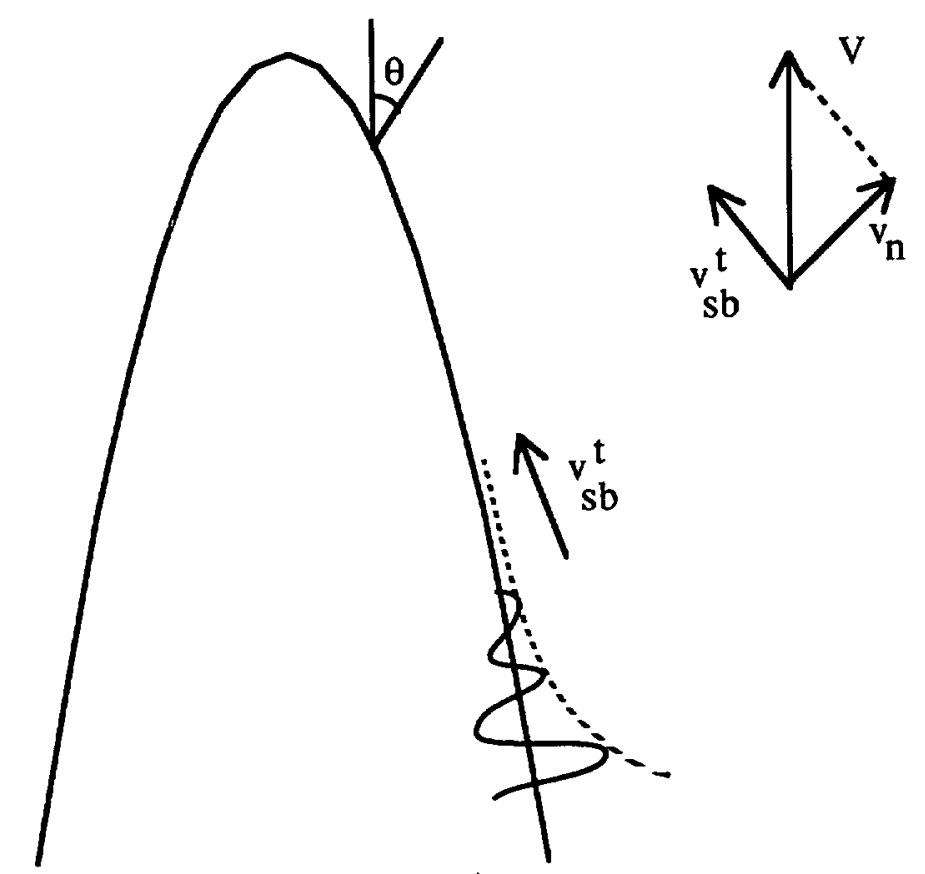

a)

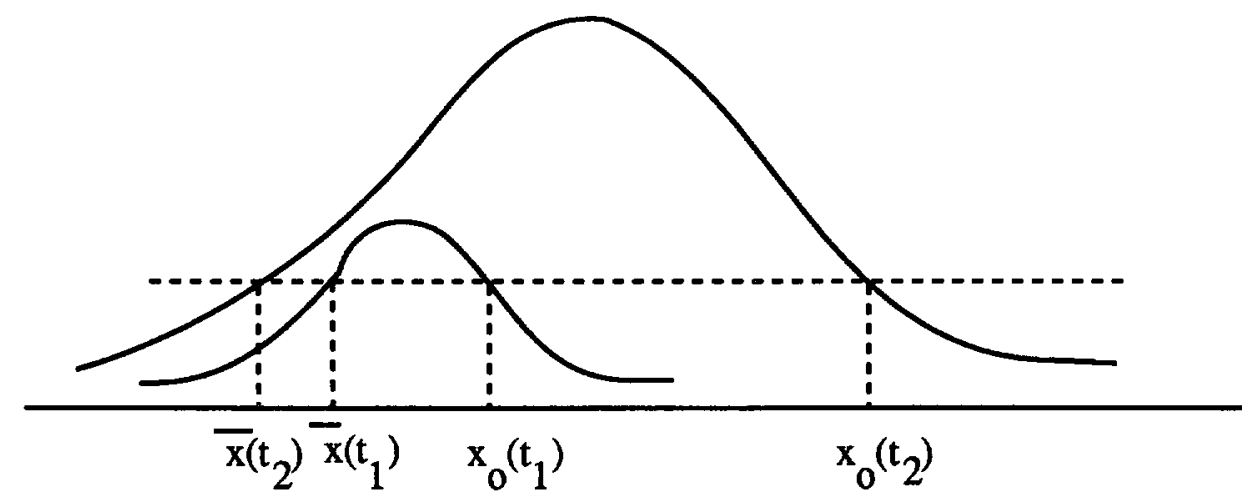

b)

Fig. 1. - (a) Sketch of a needle with sidebranch perturbations. The various velocities mentioned in the text are indicated. (b) Illustration of the fact that the evolution of a wave-packet involves amplification, spreading and advection. Note that although the center of the wave-packet, indicated by $x_{0}(t)$, moves to the right, the left position of the point $\bar{x}(t)$ at which the amplitude of the perturbation is constant moves to the left. 
The z-component $V_{s b}^{z}$ of the propagation velocity of the sidebranch front in the laboratory frame is therefore

$$
\begin{aligned}
V_{s b}^{z} & =v_{s b}^{t} \sin \theta+v_{n} \cos \theta \\
& =V \cos \theta(\sqrt{3} \sin \theta+\cos \theta) .
\end{aligned}
$$

The condition for the sidebranch front to encroach on the tip, $V_{s b}^{z}>V$, then becomes $\left(^{5}\right)$

$$
\theta<60^{\circ}
$$

According to this local zeroth-order picture, the needle crystal profile is absolutely unstable in the tip region, and convectively unstable only for $\theta>60^{\circ}$.

Of course, the distinction between absolute and convective instabilities can, strictly speaking, only be made in an infinite system. Moreover, we have neglected curvature effects, which is clearly not justified for profiles with $\lambda_{\mathrm{MS}} / \rho=\mathcal{O}(1)$. However, on the basis of what is known from calculations on similar problems in simple models with a spatially varying control parameter [19], we expect the approximation to become increasingly accurate for very small anisotropies, since then $\lambda_{M S} / \rho \rightarrow 0$. Although this analogy would suggest that there is a nonzero threshold value for the anisotropy (below which the needle profile would be unstable), this issue can only be decided on the basis of a global stability analysis.

Note that the above local description should also be relevant for non-parabolic profiles with large quasi-planar regions which seem, on the basis of computer simulations [17], [18], to be favoured by kinetic anisotropy. Indeed, such an instability of a needle crystal as a result of the tip being overtaken by the sidebranches has been found by Pieters [18] in his simulations of the boundary layer model.

We can extract from the results (4) another prediction for the propagation of a sidebranch front. If we define a local branch spacing $\lambda^{*} \equiv 2 \pi / k_{r}^{*}$, and a characteristic growth amplification length $A^{*} \equiv 1 / k_{t}^{*}$, the front propagating with the marginal stability speed $v^{*}$ is characterized by

$$
\frac{\lambda^{*}}{\Lambda^{*}}=\frac{2 \pi}{\sqrt{3}}=3.63
$$

independent of the surface tension. We will come back to this result later.

\section{Connection with the wave-packet analysis.}

The results from the previous analysis may, at first sight, appear to contradict the conclusions of the wave-packet analysis of perturbations. This apparent contradiction arises from the fact that the discussion usually focusses on the center of the packet. This center is indeed moving monotonically away from the tip, but at the same time, its amplitude is growing. In addition, the wave packet spreads. Thus, in order to make contact with the front propagation picture, one should rather study iso-deformation trajectories, i.e. the trajectories of a point at which the amplitude of the perturbation remains constant in time. As figure 1(b) illustrates, its dynamics is the net result of spreading, amplification and advection of the wave-packet, and while the center of the packet always moves away from the tip, a point of constant amplitude of the deformation may actually move towards the tip. We will see below that this does indeed happen, at least transiently.

$\left.{ }^{5}\right)$ If the sidebranch front would spread with a velocity larger than $v^{*}$, as is possible according to equation (1), the limiting angle in (8) becomes larger than $60^{\circ}$. 
Following reference [11] we describe the deformation of the needle profile $z(x)$ by $\delta z=\delta z_{0} \mathrm{e}^{S(x, t)}$, where

$$
S(x, t)=f(t)+\frac{i Q(t)}{\sqrt{\sigma} \cos \theta(t)}\left(x-x_{0}(t)\right)-\frac{A(t)}{2 \sqrt{\sigma} \cos ^{2} \theta(t)}\left(x-x_{0}(t)\right)^{2}
$$

Here lengths are measured in units of the tip radius, and time in units of $\rho / V$. The evolution of the packet is then given by the following equations (we take $x_{0}>0$ )

$$
\begin{aligned}
\dot{x}_{0} & =\sin \theta \cos \theta \\
\dot{Q} & =A\left(\cos \theta-3 Q^{2}\right) \\
\dot{A} & =-6 Q A^{2}, \\
\dot{f} & =\frac{1}{\sqrt{\sigma}} Q\left(\cos \theta-Q^{2}\right)+\dot{\theta} \tan \theta+\mathcal{O}\left(\frac{\rho V}{D} \sqrt{\sigma}\right) .
\end{aligned}
$$

This set of equations must be closed by specifying the relation between $x_{0}$ and $\theta$ which describes the unperturbed needle profile.

It should be emphasized that the Zel'dovich-like descriptions of wave packet dynamics are all only valid for very small values of $\sigma$, since the width of the packet must, on the one hand, be much smaller than the tip radius and, on the other hand, be much larger than $\lambda_{\text {MS. }}$.

Let us now follow a particular level of the perturbation amplitude, i.e. the trajectory $\bar{x}(t)$ such that $\operatorname{Re} S(\bar{x}(t), t)=-c$ (a constant), and $f(t=0)=0$. This gives for the trajectory corresponding to the side of the packet closest to the tip

$$
\bar{x}(t)=x_{0}(t)-\cos \theta(t)\left(\frac{2(f(t)+c)}{A(t) / \sqrt{\sigma}}\right)^{\frac{1}{2}}
$$

Let us first consider the case of a wave packet propagating along a planar piece of interface with a constant angle $\theta$. In this case, the wave packet analysis should reduce to the exact asymptotic results obtained for perturbations in homogeneous systems using the pinch point analysis [20]; the latter is known [15] to be equivalent to the linear marginal stability description expressed by equations (2), (4) and (5). The asymptotic regime of equations (11)(14) is in this case

$$
Q=\sqrt{\frac{\cos \theta}{3}}, \quad A=\frac{1}{6 Q t}, \quad f=2 \sigma^{-\frac{1}{2}} Q^{3} t,
$$

from which

$$
\bar{v}(t)=\dot{\bar{x}}(t) \approx \cos \theta(\sin \theta-\sqrt{8 / 3} \cos \theta) .
$$

This equation has indeed the same form as equation (7), with the minor difference that in this case the tail of the wave-packet encroaches on the tip only for angles ( ${ }^{6}$ )

$$
\theta<\arctan \sqrt{8 / 3}=58.5^{\circ}
$$

${ }^{6}{ }^{6}$ The difference between the critical angle obtained from the wave packet analysis and the previous one equation (7) can be traced back to the technical details of the approximations made to arrive at equations (11)-(14). In particular, the fact that in the regime (17) the lower limit of inequality (36) of reference [11] is violated, means that in the reduction of the front equation to a differential equation, the computation of the saddle point should be improved upon. 
We can now proceed one step further and study the iso-deformation trajectory for a parabolic profile with a large tip radius, i.e. with a small value of $\sigma$. It is clear from equations (11)-(14) that the main effect of decreasing $\sigma$ is to increase the growth rate $\dot{f}$. If we compare the evolution of $\bar{x}$ for packets with the same initial width $A(t=0) / \sqrt{\sigma}$, equation (15) shows that the smaller $\sigma$, the more $\bar{x}$ is pushed up towards the tip. As illustrated in figure 2 by the solid curves, this is confirmed by numerically integrating these equations. It is seen that for a parabolic needle profile in the small $\sigma$-range a point in the tail of the initial wave-packet always starts moving towards the tip before being finally driven away by advection. In other words, in the cases shown in figure 2 the deformation at a given distance from the tip (but close to it)

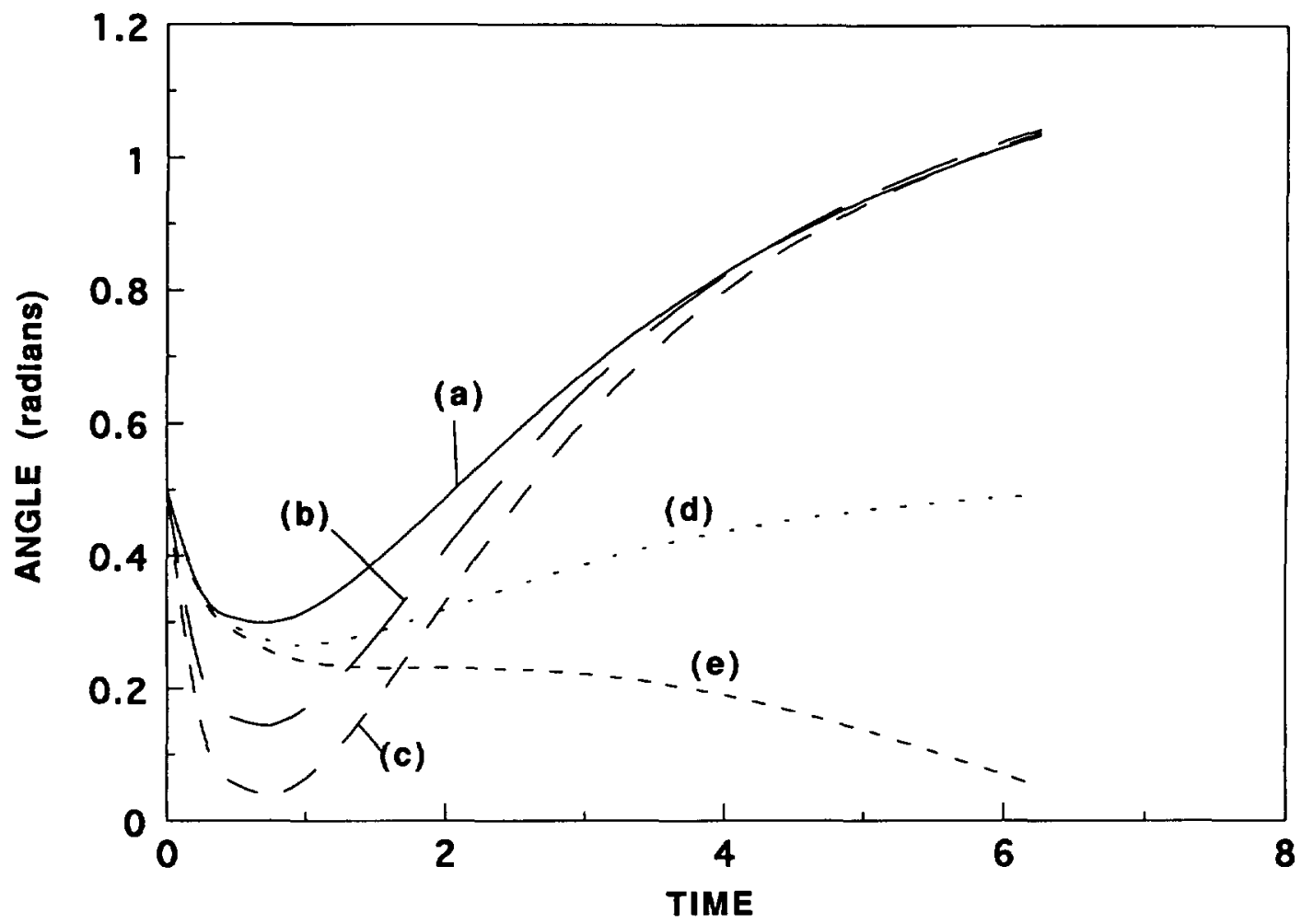

Fig. 2. - Some illustrative results for the angle $\bar{\theta}$ at the point $\bar{x}$ obtained by solving equations (11)-(14) numerically with initial conditions $\theta_{0}=0.55\left(31.5^{\circ}\right), Q=0.1, c=2, A /\left(2 \sqrt{\sigma} \cos ^{2} \theta_{0}\right)=(0.04)^{-2}$ and $\sigma=10^{-7}$ [curve (a)], $\sigma=2 \times 10^{-8}$ [curve (b)] and $\sigma=10^{-8}$ [curve (c)]. Curves (d) and (e) are for the same initial conditions as for curve (a), but with the profile given by equation (19) with $\phi=57.9^{\circ}$ for curve (d) and $\phi=56.4^{\circ}$ for curve (e).

initially increases before decaying at late times. This effect is stronger the smaller $\sigma$ is. Analogous behavior is found for different values of $c$ and values for $\sigma$ comparable to experimental ones, for which, however, as stated above, the theory is no longer reliable.

Guided by the results (7) and (17) for the planar profile, we have also computed $\bar{x}$ for profiles which interpolate between a parabolic tip and a planar tail with angle $\phi$, defined 
by the differential equation

$$
\frac{\mathrm{d} \theta}{\mathrm{d} x}=\frac{\cos ^{2} \theta-\cos ^{3} \phi / \cos \theta}{1-\cos ^{3} \phi}
$$

for the interface profile. For $\phi=90^{\circ}$, this yields a parabola. As could be expected, as $\phi$ decreases from $90^{\circ}$, the excursions of $\bar{x}$ towards the tip increase - see curves (d) and (e) of figure 2 . When $\phi$ exceeds the critical angle $58.5^{\circ}, \bar{x}$ never recedes and perturbations at the tip continue to grow indefinitely.

Thus, the study of the evolution of the tail of the wave-packet qualitatively agrees with the picture emerging from the sidebranch front approach in the previous section. Unfortunately, the Zel'dovich type of approximations which underlie the wave-packet formalism break down in the immediate vicinity of the tip; hence we can take these results only as a hint for the possibility of an instability. At this point one should therefore try to calculate directly the linear spectrum. Our attempts to do so have led us to conclude that this is impossible within the famework of the existing analytical approaches [3], [5], [11]. Indeed, these take advantage of the reduction of the linearized integral equation for profile deformations to a differential equation which, to lowest order in the curvature of the interface, yields the local MullinsSekerka dispersion relation. Solutions are then constructed by matching the solution of the inner problem close to the singularity in the complex plane to WKB outer solutions. This procedure is valid only when one can find an eigenfunction which is analytic either in the upper or lower half plane. When one imposes the conditions that ensure this analyticity, one finds that the condition for non-divergence of the eigenfunctions for $x \rightarrow \pm \infty$ can only be satisfied for a perturbation $\delta z \sim \mathrm{e}^{\omega t}$ in a wedge of the complex $\omega$ plane surrounding the positive real axis (defined by $\left.\left\{\left|\omega_{1}\right|<\min \left(\omega_{r}^{3}, \omega_{r}^{1 / 3}\right), \omega_{r}>0\right\}\right)$. While the unstable tip-splitting modes studied by Bensimon et al. [5] and Brener et al. [3] clearly satisfy this condition, neither the stable modes found numerically [7] nor a possible Hopf bifurcation can be investigated within these approximations $\left({ }^{7}\right.$ ). For such stable or neutral oscillating modes the full nonlocal problem will have to be solved in the inner region, which we have not been able to do.

Another open question emerging from our analysis is the cross-over from the wave packet analysis to the marginal stability viewpoint. According to the latter, a front that has enough time to develop in the region $\theta<60^{\circ}$ will always continue to grow towards the tip, even if nonlinearities become important. According to the wave packet analysis, however, the edges of the wave packet eventually always recede towards the sides of the needle. Possibly, nonlinearities also play a role in the cross-over of the spreading described by the wave packet analysis to the long time asymptotics described by the marginal stability analysis, but even for simple model equations, this cross-over is only poorly understood [15].

\section{Discussion.}

Although, as we have just discussed, technical difficulties prevent us from reaching definite conclusions, our results indicate that the question of stability of needle crystals and the origin of sidebranches is richer than previous results may have suggested. We now suggest some theoretical and experimental lines of investigation which could shed light on this issue.

(7) The reason why Barber et al. [10] can avoid this problem and only impose convergence of their modes on one side of the needle crystal is that they concentrate on the asymptotic behavior of the center of a wave-packet under the tacit assumption that deformations on the other side of the needle contribute exponentially small terms. 
Numerical results for the continuous spectrum are only available for a few (rather large) values of the surface tension [7] (See also Pillet [21]). At the values studied, the needle crystal is linearly stable. Although numerical methods cannot probe the regime of very small anisotropies, it would nevertheless be helpful to study how the relaxation rate of the least stable mode evolves with $\sigma$, as this could give indications about the proximity of a linear instability. Whether such an instability should occur at a nonzero values of $\sigma$ is certainly not clear, as the study of the boundary layer model has illustrated : in his simulations of this model Pieters [18] observed an instability at a finite value of the anisotropy that appears to be consistent with our scenario ; nevertheless Liu and Goldenfeld [22], who studied the linear spectrum of the needle, found no signs of a linear instability at the corresponding parameter values. This suggests that the instability might be a nonlinear one. If such is the case, a full dynamic simulation would be needed. Introducing kinetic anisotropy might help to enhance the effect through the flattening of the basic profile.

Nonlinearities could play a role in two different ways : the nonlinear coupling of the perturbation to the tip profile and curvature could be important, or nonlinearities could already play a role in determining the front propagation velocity of a front spreading along a MullinsSekerka unstable planar interface. As regards the latter possibility, we have seen in section 2 that it is known for front propagation into homogeneous unstable states that nonlinearities can only give rise to front velocities larger than $v^{*}$. Although there is no general criterion for when this happens, saturating nonlinearities generally tend to favour fronts that propagate with $v^{*}$, whereas if the nonlinearities increase the instability (e.g. near a subcritical bifurcation), fronts with $v>v^{*}$ are found [15]. In the case of the Mullins-Sekerka instability, the situation is unclear, but if the nonlinearities indeed do increase the front propagation velocity, the instability is enhanced and inherently nonlinear. Unfortunately, the paper by Pieters [18] on the boundary layer model does not provide enough information to settle this issue. An extension of his work would allow one to check directly whether the sidebranch front does indeed propagate with a velocity close to $v^{*}$ in the boundary layer model, or whether nonlinearities in the front propagation drive the velocity even larger and make the instability nonlinear.

The experiments which have been aimed at measuring the growth rate of the sidebranch amplitude as a function of distance [23], [24] have been compared with an $\mathrm{e}^{b s^{\beta}}$ dependence, with $s$ the arclength and with $\beta=1 / 4$ or $1 / 2$. A power $\beta=1 / 4$ is suggested by the wavepacket analysis. While the weak level of coherence between sidebranches on opposite sides of the dendrite profile in the experiments of Dougherty et al. [23] agrees with the noiseamplification scenario, the measurements of the spatial growth rate of the branch amplitudes in fact seem more consistent with an $\mathrm{e}^{s / \Lambda}$ variation. A front picture of sidebranch propagation would, on the other hand, naturally lead to such an exponential behavior but at the same time to a strong coherence. In such a situation, it is clear that it will be worthwhile to perform more experiments of this type on materials with various types and levels of anisotropies. In order to characterize the sidebranch behavior quantitatively, it would be useful to measure the dimensionless growth ratio

$$
r \equiv \frac{\lambda}{\Lambda}
$$

where $\lambda$ is the branch spacing and $\lambda$ the exponential growth amplification length $\left(\sim \mathrm{e}^{s / \Lambda}\right.$ ). Dougherty et al. [23] measure $\lambda \approx 16 \mu \mathrm{m}, \Lambda \approx 14 \mu \mathrm{m}$, so $r \approx 1.1$ in their experiments. If sidebranches would evolve according to the linear marginal stability predictions, then we would according to equation (9) expect a much larger ratio, namely $r=r^{*}=3.63$ (in the absence of kinetic anisotropy).

We expect that systematic experiments on different materials will reveal the following 
trend : the larger the value of the ratio $r$, the more coherent the sidebranches should be $\left(^{8}\right)$. In particular coherently oscillating dendrites are most likely to correspond to values of $r$ in the range of $r^{*}$.

\section{Acknowledgments.}

We have benefited from stimulating discussions with E. Brener. C.C. and W.v.S. gratefully acknowledge the hospitality of the Aspen Center for Physics, where part of this work was performed. The work by B.C. and C.C. is supported in part by ATP/CNES-PIRMAT 1990.

\section{References}

[1] Kessler D. A., Koplik J. and Levine H., Adv. Phys. 37 (1988) 255.

[2] LANGer J. S. in «Chance and Matler», J. Souletie, J. Vannimenus and R. Stora Ed. (NorthHolland, 1987).

[3] Brener E. A. and Mel'nikov V. I., Adv. Phys. 40 (1991) 53.

[4] See e.g. Bensimon D., Kadanoff L., Liang S., Shraiman B. I. and Tang C., Rev. Mod. Phys. 58 (1986) 977 , and references therein.

[5] Bensimon D., Pelcé P. and Shraiman B. I., J. Physique 48 (1987) 2081.

[6] Brener E. A., Iordanskil S. V. and Mel'nikov V. I., Soviet Phys.-JETP 67 (1988) 2574.

[7] Kessler D. A. and Levine H., Phys. Rev. Lett. 57 (1986) 3069.

[8] Martin O., Goldenfeld N., Phys. Rev. A 35 (1987) 1382.

[9] Zel'dovich Y. B., Istratov A. G., Kidin N. I. and Librovich V. B., Comb. Sci. Technol. 24 (1980) I.

[10] Barber M. N., Barbieri A. and Langer J. S., Phys. Rev. A 36 (1987) 3340.

[11] Caroli B., Caroli C. and Roulet B., J. Physique 48 (1987) 1423.

[12] EsIPOv S. E., Phys. Rev. A 45 (1992) 7274.

[13] Ben-Jacob E., Brand H. R., Dee G., Kramer L. and Langer J. S., Physica D 14 (1985) 348.

[14] Van SaArloos W., Phys. Rev. A 37 (1988) 211.

[15] Van SaArloss W., Phys. Rev. A 39 (1988) 6367.

[16] See e.g. Langer J. S., Rev. Mod. Phys. 52 (1980) 1.

[17] Classen A., Misbah C., Müller-Krumbhaar H. and Saito Y., Phys. Rev. A 43 (1991) 6920.

[18] Pieters R., Phys. Rev. A 37 (1988) 3126.

[19] Chomaz J. M., Huerre P. and Redekopf L. G., Phys. Rev. Lett. 60 (1988) 25.

[20] Lifshitz E. M. and Prtaevski L. P., in «Landau and Lifshitz Course of Theoretical Physics », vol. 10, Physical Kinetics (Pergamon Press);

Bers A. in « Handbook of Plasma Physics », Eds. M. N. Rosenbluth and R. S. Sagdeev (NorthHolland, Amsterdam, 1983).

[21] Pillet C.-A., Commun. Math. Phys. 140 (1991) 241, and to be published.

[22] LiU F. and Goldenfeld N., Phys. Rev. A 38 (1988) 407.

$\left(^{8}\right)$ Note in this regard that the first sidebranches of anomalous Saffman-Taylor fingers with oscillating tips and sidebranches already have very large amplitude [25]. This might suggest a large value of $r$, but unfortunately the growth seems to be so rapid and nonlinear that it seems unjustified to asign a welldefined value of $r$ to these anomalous fingers. Dendrites with oscillating tips and similar rapid growth of the sidebranch envelope have also been observed by Rolley [26] in ${ }^{3} \mathrm{He}$, but the growth conditions in this case are unclear. The lateral spreading of a front along a Mullins-Sekerka unstable interface in a HeleShaw cell has been measured and compared to the marginal stability predictions by DiFrancesco and Maher [27]. In these experiments $r$ was not estimated, but judging from the plots of the interface [28] one expects the value of $r$ to be significantly larger than one. 
[23] Dougherty A., Kaglan P. D. and Gollub J. P., Phys. Rev. Lett. 58 (1987) 1652 ;

Dougherty A. and Gollub J. P., Phys. Rev. A 38 (1988) 3043.

[24] Qian X. W. and Cummins H. Z., Phys. Rev. Lett. 64 (1990) 3038.

[25] Rabaud M., Couder Y. and Gérard N., Phys. Rev. A 37 (1988) 935.

[26] Rolley E., Ph. D. Thesis, Universite Paris VI (1989), p. 77.

[27] Difrancesco M. W. and Maher J. V., Phys. Rev. A 40 (1989) 295.

[28] Difrancesco M. W. and Maher J. V., Phys. Rev. A 39 (1989) 4710. 